\section{Remisión espontánea de acromegalia y enfermedad de Cushing en adultos mayores. Casos clínicos}

\author{
A. VERÓNICA ARAYA ${ }^{1}$, DANIELA EUGENIN ${ }^{1}$, MELCHOR LEMP $^{2}$
}

\section{Spontaneous remission of acromegaly and Cushing's disease in older patients. Report of two cases}

Pituitary diseases such as acromegaly and Cushing's disease require surgical or medical therapy. In some functioning pituitary tumors, a spontaneous remission of hormonal hypersecretion is observed, mainly associated to apoplexy or pituitary infarction. We report the evolution of two female patients older than 70 years at the time of diagnosis, with multiple comorbidities. In case 1, acromegaly was diagnosed at 74 years of age. Sellar CT scan showed a $10-\mathrm{mm}$ adenoma. During her follow-up, IGF1 levels remained normal. Nine years later, a magnetic resonance (MR) showed a 7-mm adenoma. In case 2, clinical and biochemical diagnosis of Cushing's disease was done being 71 years old. Sellar MR showed a 6-mm adenoma. Three years later, urinary cortisol normalized with no changes in adenoma at MR. Seven years later, she remains without clinical or biochemical signs of hypercortisolism. In both cases, no signs of hemorrhage were observed at $M R$.

(Rev Med Chile 2018; 146: 933-937)

Key words: Acromegaly; Cellular Senescence; Pituitary ACTH Hypersecretion; Remission, Spontaneous.
'Sección Endocrinología y

Diabetes, Hospital Clínico de la Universidad de Chile, Santiago,

Chile.

${ }^{2}$ Departamento de Neurología y Neurocirugía, Hospital Clínico de la Universidad de Chile. Santiago, Chile.

Los autores declaran no tener conflictos de interés.

Trababo no recibió

financiamiento.

Recibido el 30 de enero de 2018, aceptado el 17 de julio de 2018.

Correspondencia a:

Dra. Verónica Araya

Sección Endocrinología y

Diabetes, Hospital Clínico de la

Universidad de Chile, Santiago,

Chile.

Santos Dumont 999, Santiago,

Chile.

varaya@hcuch.cl
$\mathrm{P}$ atologías hipofisiarias como la acromegalia, determinada por un tumor productor de hormona de crecimiento (GH) y la enfermedad de Cushing, determinada por un tumor productor de ACTH, requieren de una terapia quirúrgica, médica o ambas para la resolución del cuadro clínico. En las últimas 4 décadas, se han descrito en la literatura casos de tumores hipofisiarios funcionantes que presentaron una remisión espontánea de la hipersecreción hormonal en algún momento de su evolución. La gran mayoría de ellos corresponde a tumores mayores de $1 \mathrm{~cm}$ o macroadenomas que presentaron una apoplejía hipofisiaria, cuadro que, entre otras complicaciones, puede determinar un hipopituitarismo, es decir, una disminución de la secreción de las hormonas reguladas por la hipófisis. Entre los casos descritos hay productores de $\mathrm{GH}$ y, en menor número, tumores productores de $\mathrm{ACTH}^{1-7}$.

Presentamos dos casos de pacientes mayores de 70 años al momento del diagnóstico de acromegalia y enfermedad de Cushing. Ambos tumores correspondían a microadenomas, que no fueron operados ni tratados con terapia farmacológica, observándose en el tiempo una tendencia a la normalización de los niveles hormonales, sin cambios significativos en la imagen tumoral ni en la función de los otros ejes hipofisiarios.

\section{Caso 1}

Paciente de sexo femenino, con historia de asma bronquial, hipertensión arterial, hipertrofia miocárdica septal, obesidad e hipotiroidismo de 
Remisión espontánea de acromegalia y enfermedad de Cushing - V. Araya et al

larga data. A los 74 años, en control médico se pesquisa facies acromegaloide, manos anchas con dedos gruesos y pies grandes. Se solicitó GH basal que estaba elevada: 2,8 y $1,6 \mathrm{ng} / \mathrm{ml}$. Posteriormente se realizó medición postcarga de $75 \mathrm{~g}$ de glucosa, observándose a los 60 min un valor de GH que no suprimió: $2,3 \mathrm{ng} / \mathrm{ml}$ con glicemia de $166 \mathrm{mg} / \mathrm{dl}$. Hormonas tiroideas bajo terapia de sustitución con levotiroxina en rango normal (T4L: $1,49 \mathrm{ng} /$ dl, TSH: $1,45 \mathrm{mUI} / \mathrm{L}$ ) y prolactina (PRL): 22,9 $\mathrm{ng} / \mathrm{ml}$. Se solicitó una tomografía computada (TAC) de silla turca que evidenció un adenoma de $10 \mathrm{~mm}$ lateralizado a izquierda y desviación del tallo a derecha. La paciente rechazó tratamiento quirúrgico. Tres años después se realizó nueva TAC de silla turca que mostró adenoma de $7 \mathrm{~mm}$ que deformaba el diafragma selar y provocaba un leve hundimiento de la silla turca, sin signos de hemorragia intratumoral. En ese momento, el IGF 1 (factor de crecimiento insulino-simil 1) se encontraba en rango normal bajo para la edad: $64,1 \mathrm{ng} / \mathrm{ml}(64-188 \mathrm{ng} / \mathrm{ml})$. A los siete años de seguimiento, se realizó nueva medición de GH postcarga de glucosa, a los 60 min se observó un valor de $1 \mathrm{ng} / \mathrm{ml}$ con glicemia de $174 \mathrm{mg} / \mathrm{dl}$. A los nueve años se reevaluó con resonancia nuclear magnética (RM), que mostró presencia del adenoma de $7 \mathrm{~mm}$, lateralizado a izquierda, sin signos de hemorragia (Figura 1) y medición de IGF1 bajo el rango normal: $48,1 \mathrm{ng} / \mathrm{ml}(55-166 \mathrm{ng} / \mathrm{ml})$.

\section{Caso 2}

Paciente de sexo femenino, con historia de obesidad, diabetes mellitus tipo 2, hipertensión arterial (HTA), enfermedad pulmonar crónica asociada a tabaquismo e hipotiroidismo subclínico. A los 71 años, se evaluó por mal control de HTA y diabetes mellitus. Al examen físico se observaba facies cushingoide y relleno de huecos supraclaviculares. Se solicitó cortisol libre urinario (CLU): 236 ug/24 h (VN hasta $100 \mathrm{ug} / 24 \mathrm{~h}$ ), test de Nugent o de supresión nocturna con dexametasona (Dx) 1 mg: 11,1 ug/dl (VN < 1,8 ug/dl), ACTH 32,1pg/ml (VN hasta $46 \mathrm{pg} / \mathrm{ml}$ ). Se realizó estudio funcional: test de $\mathrm{Dx} /$ desmopresina, que mostró respuesta de cortisol postestímulo, sugiriendo origen hipofisiario. Se solicitó RM de silla turca que demostró adenoma posterolateral izquierdo de $6 \mathrm{~mm}$, que determina leve remodelación del piso selar (Figura 2A). Por distintas razones, la paciente no se realizó cirugía. En controles sucesivos se observó normalización del CLU sin mediar tratamiento. A los 3 años tuvo un test de Nugent de 3,5 ng/dl, ACTH: $54,8 \mathrm{pg} / \mathrm{ml}$ y CLU: 50,1 ug/24 h. Una nueva RM de silla turca mostró adenoma de similares características, sin evidencias de hemorragia intratumoral (Figura 2B). A los 7 años tiene CLU 28,8 ug/24 h, la diabetes mellitus mantiene un control adecuado para la edad (HbAlc: 7,6\%) y ha disminuido 10 kilos de peso desde su evaluación inicial.
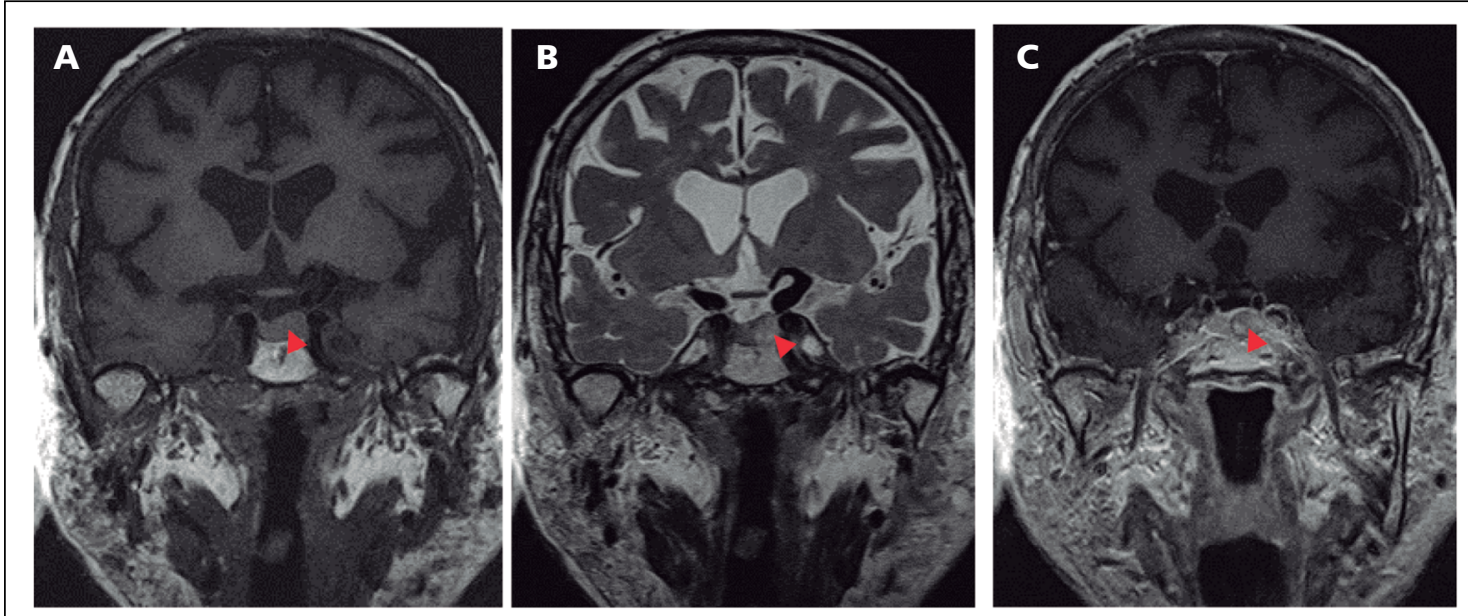

Figura 1. Cortes coronales en resonancia nuclear magnética de silla turca realizada 9 años después del diagnóstico de acromegalia. En el estudio sin contraste, la flecha indica un nodulo de $7 \mathrm{~mm}$, lateralizado a izquierda, isointenso en secuencia T1 (A) e hiperintenso en secuencia T2 (B). Después de inyección con gadolineo, se observa leve realce del nódulo en secuencia T1 (C). 

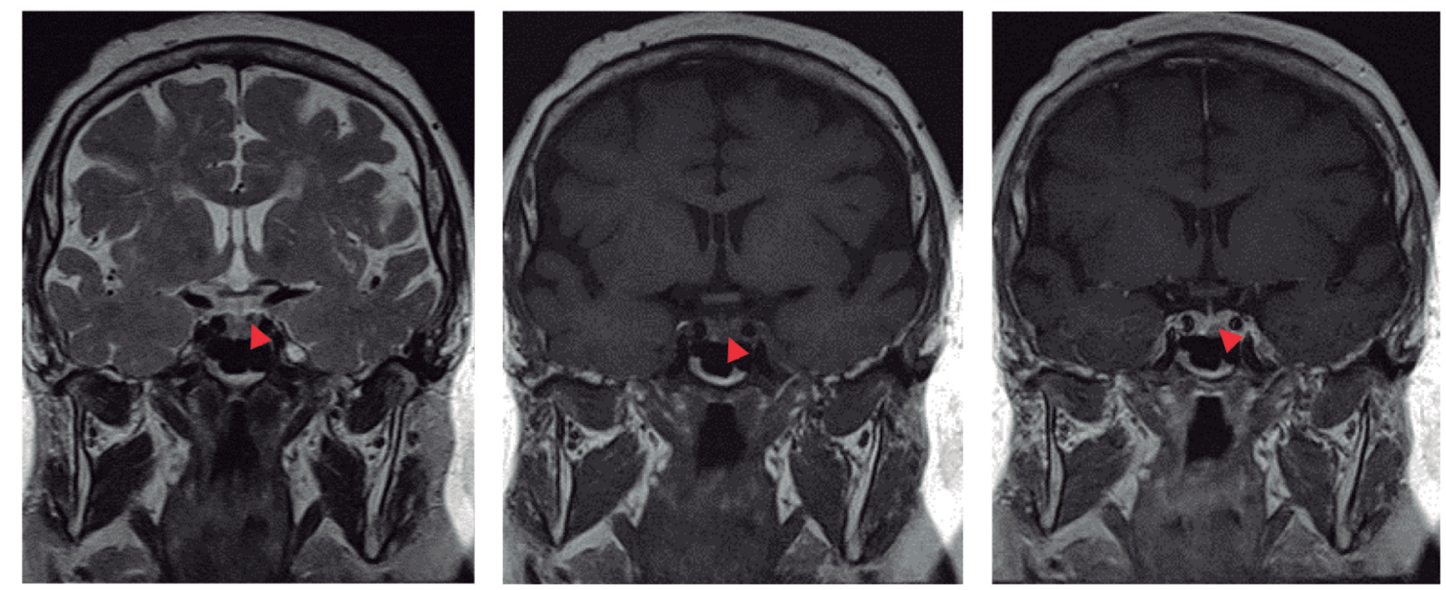

Figura 2A. Cortes coronales en resonancia nuclear magnética de silla turca al momento del diagnóstico, en el estudio sin contraste la flecha indica un nódulo de $6 \mathrm{~mm}$, posterolateral izquierdo, isointenso en secuencias T1 y T2 (recuadros izquierdo y central). Después de la administración de gadolinio, realza escasamente al medio de contraste. La imagen hiperintensa en T1 adyacente al piso selar, corresponde a estructura vascular venosa (recuadro derecho).
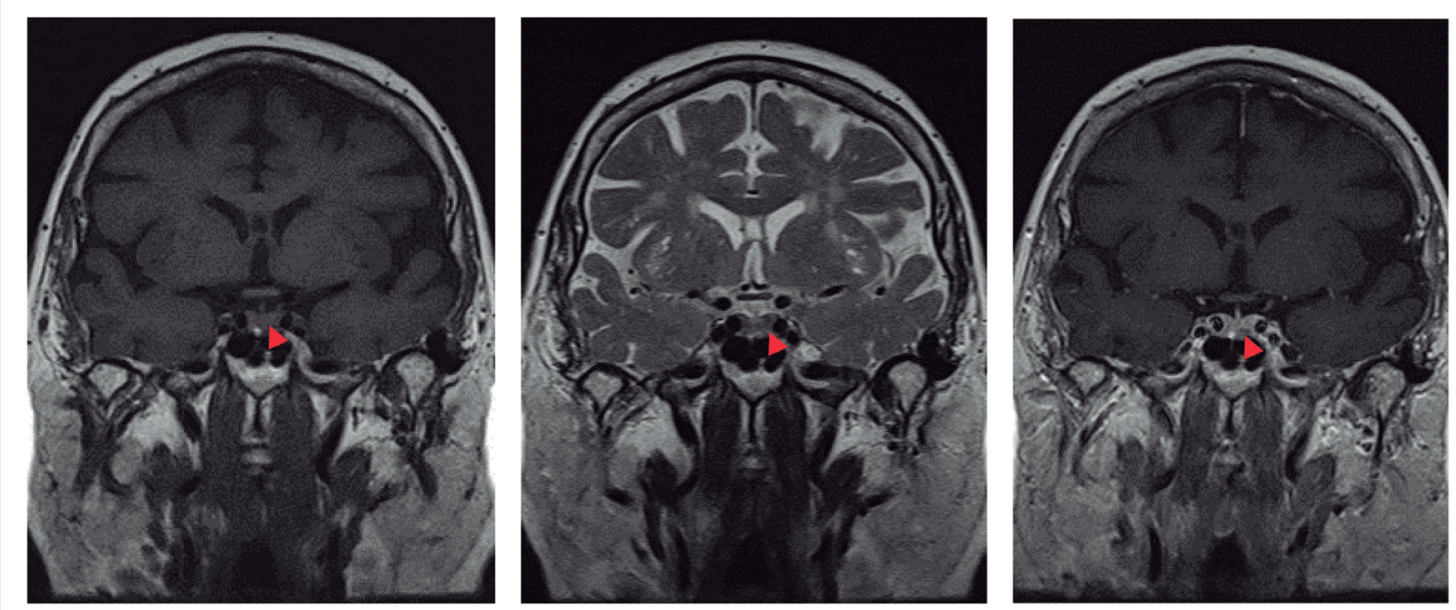

Figura 2B. Control de resonancia nuclear magnética de silla turca a los 3 años. En cortes coronales, en el estudio sin contraste se observa el nódulo, posterolateral izquierdo, isointenso en secuencias T1 y T2 (recuadros izquierdo y central). Se observa que realza escasamente después de la administración de gadolinio (recuadro derecho).

Ambas pacientes aún sobreviven, a los 82 y 88 años, respectivamente y no manifiestan clínica de hiperfunción endocrina.

\section{Discusión}

La mayoría de los casos descritos en la literatura en que ocurrió remisión espontánea de la hiperfunción en pacientes con acromegalia o enfermedad de Cushing están asociados a una apoplejía hipofisiaria. Casi todos estos pacientes presentaron el cuadro clínico característico de cefalea, alteraciones visuales y oftalmoplejia. En enfermedad de Cushing se han observado casos aislados de infarto del adenoma ${ }^{8-10}$. Ambos cuadros se acompañan de signos de hipopituitarismo $\mathrm{y}$, excepcionalmente, el resto de la función hipo- 
fisiaria se mantiene normal ${ }^{5}$. En nuestros casos observamos un descenso de los niveles hormonales sin manifestaciones de hipopituitarismo. Además, estas pacientes tenían adenomas pequeños, a diferencia de los casos de apoplejía hipofisiaria que generalmente corresponden a macroadenomas.

Los tumores hipofisiarios en su gran mayoría son lesiones pequeñas, de crecimiento lento y habitualmente corresponden a neoplasias benignas.

Se ha atribuido esta característica a que en las células tumorales ocurre un envejecimiento precoz o muerte celular programada ${ }^{11}$.

El envejecimiento consiste en una señal de transducción que lleva a una detención del ciclo celular seguido de cambios en el fenotipo de la célula. Por otra parte, esto lleva a una detención de la proliferación celular, pero permite que esta mantenga sus funciones fisiológicas y continúe viable.

Se ha demostrado que los adenomas hipofisiarios, sobre todo los productores de GH, presentan alteraciones en el gen Pttg (pituitary tumor transforming gene), consistentes en deleciones o sobreexpresión, las que llevan a inestabilidad de cromosomas, aneuploidía y daño del ADN. Además, Pttg facilita la expresión de una proteína que promueve el envejecimiento de células tumorales, la cyclin-dependent kinase (CDK) inhibitor $p 21^{12-14}$.

Por otra parte, existen adenomas productores de GH escasamente granulares, que se caracterizan por menor secreción hormonal, pero, habitualmente, estos son más frecuentes en sujetos jóvenes y corresponden a macroadenomas invasores, lo que no concuerda con nuestro caso ${ }^{15}$. Se ha observado en estos tumores una menor expresión de las proteínas SA- $\beta$-GAL (senescence associated lysosomal $\beta$-galactosidase) y ciclina $\mathrm{p} 16$, también relacionadas con envejecimiento celular, lo que explicaría su comportamiento más agresivo ${ }^{16}$.

Una explicación plausible a lo observado en nuestro caso es que los mecanismos de senescencia celular, presentes naturalmente en adenomas hipofisiarios, podrían ser acentuados por el envejecimiento fisiológico, lo que favorecería en pacientes ancianos la resolución espontanea de la hipersecreción hormonal, sin afectar el normal funcionamiento de la hipófisis.

En relación a la enfermedad de Cushing, generalmente ocurre por la presencia de microadenomas, que suelen ser más agresivos desde el punto de vista funcional y clínico, pero que no muestran un nivel elevado de proliferación celular. Respecto de los mecanismos moleculares que llevan a la formación de tumores derivados de células corticotropas y que determinan un hipercortisolismo, aún no están bien precisados. Las mutaciones génicas encontradas en estos tumores, generalmente, corresponden a mutaciones somáticas de factores de transcripción ${ }^{17}$. Un estudio observó que las células corticotropas tumorales muestran una menor expresión que las normales de SA- $\beta$-GAL y de la ciclina p27, proteína que también actúa disminuyendo la progresión del ciclo celular ${ }^{16,18}$.

Se han publicado casos aislados de remisión espontánea de enfermedad de Cushing, algunos asociados a un infarto tumoral. En estos casos generalmente se observan signos característicos en las imágenes y manifestaciones de hipocortisolismo ${ }^{2,7-10}$. Recientemente, algunos autores publicaron una serie de casos en que se observó resolución espontánea del hipercortisolismo en 2 de 132 adenomas funcionantes productores de $\mathrm{ACTH}^{19}$. Esta transformación a un estado silente pudiera explicarse, en parte, por la secreción de una forma de ACTH con menor actividad biológica. En nuestro caso observamos que a los 3 años de seguimiento había evidencia de hiperactividad del eje suprarrenal, ya que en el test de Nugent no suprimió y la ACTH se encontraba sobre el rango normal. Sin embargo, a diferencia del hipercortisolismo cíclico, en nuestro caso se observó una disminución progresiva del hipercortisolismo clínico, con normalización del CLU en mediciones repetidas, evolución que concuerda con lo expuesto previamente. Por otra parte, los mecanismos enzimáticos que llevan a la liberación de la ACTH desde la molécula de pro-opiomelanocortina (POMC) pudieran verse afectados con el envejecimiento ${ }^{20}$.

En suma, de acuerdo a la evolución observada en estas pacientes, que a lo largo de los años presentaron una remisión clínica y de laboratorio de la hipersecreción hormonal, planteamos que los mecanismos de senescencia que se encuentran naturalmente presentes en los adenomas hipofisiarios, pueden favorecer la remisión espontánea de la hiperfunción. Estos mecanismos pueden ser potenciados por el envejecimiento fisiológico en adultos mayores de 70 años con microadenomas. Por lo tanto, en ancianos con múltiples comor- 
bilidades o frágiles sería necesario evaluar cuidadosamente la conducta terapéutica a seguir, considerando que en la actualidad existen opciones de terapia médica con efectividad comprobada.

\section{Referencias}

1. Lindholm J, Bjerre P, Riishede J, Gyldensted C, Hagen C. Pituitary function in patients with evidence of spontaneous disappearance of a pituitary adenoma. Clin Endocrinol (Oxf) 1983; 18: 599-603.

2. Thomas N, Simon R, Chacko G, Chacko AG, Chandy MJ, Seshadri MS. Regression of acromegaly following pituitary apoplexy. Neurol India 1999; 47 (2): 161-2.

3. Nishioka H, Haraoka J, Miki T. Spontaneous remission of functioning pituitary adenomas without hypopituitarism following infarctive apoplexy: two case reports. Endocr J 2005; 52: 117-23.

4. Fraser LA, Lee D, Cooper P, Van Uum S. Remission of acromegaly after pituitary apoplexy: case report and review of literature. Endocr Pract 2009; 15: 725-31.

5. Roerink SH, van Lindert EJ, van de Ven AC. Spontaneous remission of acromegaly and Cushing's disease following pituitary apoplexy: Two case reports. Neth J Med. 2015; 73: 242-6.

6. Ishibashi M, Shimada K, Abe K, Furue H, Yamaji T. Spontaneous remission in Cushing's disease. Arch Intern Med 1993; 153: 251-5.

7. Araya V, Solís I, Oviedo S, Lemp M. Remisión parcial del hipercortisolismo en una Enfermedad de Cushing posterior a una apoplejía hipofisiaria. Caso clínico. Rev Med Chile 1998; 126: 1497-501.

8. Dickstein G, Arad E, Shechner C. Late complications in remission from Cushing disease. Recurrence of tumor with reinfarction or transformation into a silent adenoma. Arch Intern Med 1997; 157: 2377-80.

9. Pignatta AB, Díaz AG, Gómez RM, Bruno OD. Spontaneous remission of Cushing's disease after disappearance of a microadenoma attached to the pituitary stalk. Pituitary 2004; 7: 45-9.

10. Alarifi A, Alzahrani AS, Salam SA, Ahmed M, Kanaan
I. Repeated remissions of Cushing's disease due to recurrent infarctions of an ACTH-producing pituitary macroadenoma. Pituitary 2005; 8: 81-7.

11. Sapochnik M, Nieto L, Fuertes M, Arzt E. Molecular mechanisms underlying pituitary pathogenesis. Biochem Genet 2016; 54: 107-19.

12. Chesnokova V, Melmed S. Pituitary senescence: The evolving role of Pttg. Mol Cell Endocrinol 2010; 326: 55-9.

13. Chesnokova V, Zonis S, Kovacs K, Ben-Shlomo A, Wawrowsky K, Bannykh S, et al. p21 (Cip1) restrains pituitary tumor growth. Proc Natl Acad Sci USA 2008; 105: 17498-503.

14. Chesnokova V, Zhou C, Ben-Shlomo A, Zonis S, Tani Y, Ren SG, et al. Growth hormone is a cellular senescence target in pituitary and nonpituitary cells. Proc Natl Acad Sci USA 2013; 110: E3331-9.

15. Obari A, Sano T, Ohyama K, Kudo E, Qian ZR, Yoneda A, et al. Clinicopathological features of growth hormone-producing pituitary adenomas: Difference among various types defined by cytokeratin distribution pattern including a transitional form. Endocr Pathol 2008; 19: 82-91.

16. Manojlovic-Gacic E, Skender-Gazibara M, Popovic V, Soldatovic I, Boricic N, Raicevic S, et al. Oncogene-induced senescence in pituitary adenomas-an immunohistochemical study. Endocr Pathol 2016; 27: 1-11.

17. Theodoropoulou M, Reincke M, Fassnacht M, Komada M. Decoding the genetic basis of Cushing's disease: USP8 in the spotlight. Eur J Endocrinol 2015; 173: M73-M83.

18. Lidhar K, Korbonits M, Jordan S, Khalimova Z, Kaltsas $\mathrm{G}, \mathrm{Lu} \mathrm{X}$, et al. Low expression of the cell cycle inhibitor p27Kip1 in normal corticotroph cells, corticotroph tumors, and malignant pituitary tumors. J Clin Endocrinol Metab 1999; 84: 3823-30.

19. Zoli M, Faustini-Fustini M, Mazzatenta D, Marucci G, De Carlo E, Bacci A, et al. ACTH adenomas transforming their clinical expression: report of 5 cases. Neurosurg Focus. 2015; 38: E15-E24.

20. Cooper O. Silent corticotroph adenomas. Pituitary 2015; 18: 225-31. 